\title{
Electronic thermal conductivity of disordered metals
}

\author{
Roberto Raimondi and Giorgio Savona \\ Dipartimento di Fisica "E. Amaldi," Università di Roma Tre, Via della Vasca Navale 84, 00146 Roma, Italy \\ Peter Schwab and Thomas Lück \\ Institut für Physik, Universität Augsburg, 86135 Augsburg Germany
}

(Dated: September 7, 2018)

\begin{abstract}
We calculate the thermal conductivity of interacting electrons in disordered metals. In our analysis we point out that the interaction affects thermal transport through two distinct mechanims, associated with quantum interference corrections and energy exchange of the quasi particles with the electromagnetic environment, respectively. The latter is seen to lead to a violation of the Wiedemann-Franz law. Our theory predicts a strong enhancement of the Lorenz ratio $\kappa / \sigma T$ over the value which is predicted by the Wiedemann-Franz law, when the electrons encounter a large environmental impedance.

PACS numbers: 72.15.Eb
\end{abstract}

The Wiedemann-Franz law relates the electronic thermal conductivity, $\kappa$, and the electrical conductivity, $\sigma$, and states that the Lorenz ratio $L=\kappa / \sigma T$ is a universal constant given by $L=\pi^{2} k_{B}^{2} / 3 e^{2}$. In this equation $k_{B}$ is the Boltzmann constant, $-e$ the electron charge, and $T$ the temperature. The validity of the WiedemannFranz law relies mainly on a single-particle description of the transport properties, on the Fermi statistics of the charge carriers and on the assumption of purely elastic scattering $\stackrel{1}{\underline{1}}$. In a Fermi-liquid, one expects that this law still holds at low enough temperature, when the quasiparticles cannot exchange energy during collisions 2 . Deviations from the Wiedemann-Franz law as recently observed in the normal state of a copper-oxide superconductor have thus been interpreted as an evidence for the breakdown of Fermi-liquid theory ${ }^{3}$.

The effects of Coulomb interaction on the electrical transport at low temperature can be, broadly, grouped in two main types. From one side, transport implies adding charges to a conductor. This has an energy cost that depends on the size and shape of the conductor itself. For example in tunnel junctions the energy transfer between quasi-particles and the electrodynamical environment causes the Coulomb blockade phenomena. On the other side Coulomb interaction leads in a disordered conductor to an additional source of random scattering that interferes with the scattering from the impurities. This is a quantum effect and depends on the details of charge diffusion.

In the 1980s these quantum interferences were shown to lead to corrections to the electrical conductivity beyond the standard Fermi-liquid results. It turned out that these corrections may, in fact, be incorporated into a scale-dependent renormalization of the Landau Fermi-liquid parameters 4.5.6.7.8.9. Whithin this framework, Castellani and co-workers $\frac{10}{10}$ demonstrated that the scale-dependent corrections to the thermal conductivity are the same as the corrections to the electrical conductivity. This led them to conclude that the WiedemannFranz law is valid up to the metal-insulator transition.
This conclusion was challenged by Livanov et al. 11 who found for a two-dimensional system with long range Coulomb interaction additional contributions to the thermal conductivity. Recently, the issue has been reexamined by Niven and Smith ${ }^{12}$, who also concluded that the Wiedemann-Franz law is violated.

In this paper we study the problem by means of the quasiclassical Green's function approach, which has proved to be a powerful tool in describing the dynamical properties of superconductors 13 and the transport in hybrid mesoscopic structures 14 . Recently it was further demonstrated that both Coulomb blockade phenomena and quantum interference corrections to the charge transport can be conveniently described within this theoretical framework 15 . Advantages of the method are that it is not restricted to the linear response regime, and often provides more compact derivations than the standard diagrammatic techniques.

A perturbative calculation of the thermal conductivity, besides confirming Ref ${ }^{12}$, allows us to clarify the origin of the apparent discrepancies in the literature. To do so we separate the different physical mechanisms by their different range of exchanged energies and relevant length scales. For instance, the quantum interference effects occur over distances from the mean free path up to the thermal diffusion length $\sqrt{\hbar D / k_{B} T}$, and imply energy exchanges larger than the temperature $T$. These yield corrections which are logarithmically divergent and can be readily related to the scale-dependent renormalization of the electrical conductivity. Here the temperature acts as an infrared cutoff. The interaction effects responsible for the deviations from the Wiedemann-Franz law are associated with the long-range part of the Coulomb interaction and their singular behavior has the temperature as the upper cutoff. We successively concentrate on the long-range part of the Coulomb interaction. In particular we predict a sizable enhancement of the Lorenz ratio when the sheet resistance is of the order $h / e^{2}$ in the case of a two-dimensional electron system, and a strong enhancement of the Lorenz ratio for thin metallic wires 
when the total resistance of the wire is larger than $h / e^{2}$.

From now on we use units where $\hbar=k_{B}=1$, but we put back the constants in the final results. We start with a brief introduction of the quasiclassical formalism. For a more detailed description we defer the reader to Ref 16 . Whithin this formalism the short-distance behavior of the electron Green's function is taken into account in an averaged way from the outset by introducing the quasiclassical Green's function, which solves the Eilenberger equation ${ }^{17}$

$\left[\partial_{t_{1}}+\partial_{t_{2}}+v_{F} \hat{\mathbf{p}} \cdot \partial_{\mathbf{x}}\right] \check{g}_{t_{1} t_{2}}(\mathbf{x}, \hat{\mathbf{p}})=-\mathrm{i}[\check{\Sigma}(\mathbf{x}, \hat{\mathbf{p}}), \check{g}(\mathbf{x}, \hat{\mathbf{p}})]$

In contrast to the Dyson equation for the ordinary Green's function, the Eilenberger equation for $\check{g}$ is homogeneous and requires a normalization condition, which can be chosen of the form $\check{g} \check{g}=\check{1}$. The Green's function has a two-by-two matrix structure in Keldysh space,

$$
\check{g}=\left(\begin{array}{cc}
g^{R} & g^{K} \\
0 & g^{A}
\end{array}\right) .
$$

Matrix products imply both summation and integration over Keldysh indices and time variables, respectively. We recall that, whereas the diagonal components of $\check{g}$ describe the spectral properties of the system, the offdiagonal Keldysh component carries information about the distribution function. In this respect, the Keldysh component of Eq. (11) is the quantum analog of the Boltzmann equation.

Impurity scattering is introduced by means of the standard white-noise random potential and is described by the self-energy in the self-consistent Born-approximation as

$$
\check{\Sigma}_{t_{1} t_{2}}(\mathbf{x})=-\frac{\mathrm{i}}{2 \tau} \int \frac{d \hat{\mathbf{p}}}{\Omega_{d}} \check{g}_{t_{1} t_{2}}(\mathbf{x}, \hat{\mathbf{p}}),
$$

where $\tau$ is the elastic scattering time and $\Omega_{d}$ is the $d$ dimensional solid angle.

The charge and heat current densities have the form

$$
\left(\begin{array}{c}
\mathbf{j}_{e}(\mathbf{x}, t) \\
\mathbf{j}_{Q}(\mathbf{x}, t)
\end{array}\right)=-\frac{N_{0}}{2} v_{F} \int \frac{d \hat{\mathbf{p}}}{\Omega_{d}} \hat{\mathbf{p}} \int d \epsilon\left(\begin{array}{c}
-e \\
\epsilon
\end{array}\right) g^{K}(\mathbf{x}, t ; \hat{\mathbf{p}}, \epsilon),
$$

where $N_{0}$ is the density of states at the Fermi energy, $t=\left(t_{1}+t_{2}\right) / 2$ and $\epsilon$ corresponds to the Fourier transform of the relative time $t_{1}-t_{2}$.

In the dirty limit, the variation of the Green's function is on space and time scales larger than the elastic mean free path $l=v_{F} \tau$ and scattering time $\tau$, respectively. In this limit one may expand the Green's function $\check{g}$ in spherical harmonics and keep the $s$ - and $p$-wave components only, $\check{g}(\hat{\mathbf{p}})=\check{g}_{s}+\hat{\mathbf{p}} \check{g}_{p}+\ldots$ The Eilenberger equation is then replaced by $\left(D=v_{F}^{2} \tau / d\right)$ the Usadel equation ${ }^{18}$,

$$
\partial_{t} \check{g}_{s}(\mathbf{x})-D \partial_{\mathbf{x}}\left[\check{g}_{s} \partial_{\mathbf{x}} \check{g}_{s}\right]=0
$$

which is the analogous of the saddle-point condition in the non-linear $\sigma$-model matrix field theory $\underline{\underline{6}}$. As a result, the currents are expressed in terms of the $s$-wave component of the Green's function:

$$
\begin{aligned}
\mathbf{j}_{e}(\mathbf{x}, t) & =-\frac{e N_{0} D}{2} \int d \epsilon\left(\check{g}_{s} \partial_{\mathbf{x}} \check{g}_{s}\right)^{K}, \\
\mathbf{j}_{Q}(\mathbf{x}, t) & =\frac{N_{0} D}{2} \int d \epsilon \epsilon\left(\check{g}_{s} \partial_{\mathbf{x}} \check{g}_{s}\right)^{K} .
\end{aligned}
$$

As a simple application of the formalism we derive the Drude formula for the electrical and thermal conductivity. In the absence of interactions the Green's function $\check{g}$ reads:

$$
\check{g}_{s}(\mathbf{x}, t ; \epsilon)=\left(\begin{array}{cc}
1 & 2 F \\
0 & -1
\end{array}\right) .
$$

Near local equilibrium with a local temperature $T(\mathbf{x})$ and chemical potential $\mu(\mathbf{x})$ the function $F$ is given by

$$
F=\tanh \left(\frac{\epsilon-\mu(\mathbf{x})}{2 T(\mathbf{x})}\right)
$$

from which the Drude expressions for both electrical and heat currents are found

$$
\begin{aligned}
\mathbf{j}_{e} & =2 e^{2} D N_{0}(\nabla \mu / e) \\
\mathbf{j}_{Q} & =\frac{\pi^{2}}{3} k_{B}^{2} 2 N_{0} D T(-\nabla T),
\end{aligned}
$$

and in particular the Wiedemann-Franz law holds.

To include the effects of Coulomb interaction, we introduce ${ }^{15}$ a Hubbard-Stratonovich matrix field

$$
\check{\phi}=\left(\begin{array}{ll}
\phi_{1} & \phi_{2} \\
\phi_{2} & \phi_{1}
\end{array}\right)
$$

whose fluctuations describe the retarded, advanced, and Keldysh components of the screened Coulomb interaction

$$
-\mathrm{i} e^{2}\left(\begin{array}{cc}
\left\langle\phi_{1} \phi_{1}\right\rangle & \left\langle\phi_{1} \phi_{2}\right\rangle \\
\left\langle\phi_{2} \phi_{1}\right\rangle & \left\langle\phi_{2} \phi_{2}\right\rangle
\end{array}\right)=\frac{1}{2}\left(\begin{array}{cc}
V^{K} & V^{R} \\
V^{A} & 0
\end{array}\right) .
$$

In the presence of the field $\check{\phi}$, one first adds a term ie $[\check{\phi}, \check{g}]$ to the right-hand side of the Eilenberger (11) or the Usadel equation (5). Secondly, the resulting solution $\check{g}[\phi]$ is averaged over the fluctuations of $\check{\phi}$ according to Eq. (13). In analogy to the non-interacting case (Cf. Eq. (8)), one can define the distribution function in the presence of interactions via the relation between the Keldysh and the retarded, advanced components of the Green's function $\left\langle g^{K}\right\rangle=\left\langle g^{R}\right\rangle F-F\left\langle g^{A}\right\rangle$. We further assume a system which is - with the exception of a weak temperature gradient - translational invariant. Then it is convenient to expand the distribution function as

$$
F_{\epsilon-\omega}\left(\mathbf{x}_{1}\right) \approx F_{\epsilon-\omega}(\mathbf{x})-\partial_{T} F_{\epsilon-\omega}(\mathbf{x}) \nabla T \cdot\left(\mathbf{x}_{1}-\mathbf{x}\right)
$$

and to Fourier transform from real to momentum space. The correction to the thermal current is finally obtained as $\delta \mathbf{j}_{Q}=\delta \mathbf{j}_{Q}^{a}+\delta \mathbf{j}_{Q}^{b}$ with

$$
\begin{aligned}
\delta \mathbf{j}_{Q}^{a}= & D N_{0} \nabla T \int d \epsilon \epsilon \int \frac{d \omega}{2 \pi} \partial_{T}\left(F_{\epsilon-\omega}(\mathbf{x}) F_{\epsilon}(\mathbf{x})\right) \\
& \times \operatorname{Im} \sum_{q} \frac{1}{\left(-\mathrm{i} \omega+D q^{2}\right)^{2}} V_{\omega}^{R}(\mathbf{q})
\end{aligned}
$$


and

$$
\begin{aligned}
\delta \mathbf{j}_{Q}^{b}= & D N_{0} \nabla T \int d \epsilon \epsilon \int \frac{d \omega}{2 \pi} F_{\epsilon}(\mathbf{x}) \partial_{T} F_{\epsilon-\omega}(\mathbf{x}) \\
& \times \frac{4}{d} \operatorname{Im} \sum_{q} \frac{D q^{2}}{\left(-\mathrm{i} \omega+D q^{2}\right)^{3}} V_{\omega}^{R}(\mathbf{q}),
\end{aligned}
$$

where $d$ is the dimension of the system under consideration. Our result, Eqs. (1516), for the thermal current is equivalent to the thermal conductivity found in ${ }^{12}$ by using the diagrammatic method and the Matsubara technique. We notice that the diffusive pole appearing in Eqs. (15,16) originates from the Usadel equation (15).

Using the relation $F_{\epsilon} F_{\epsilon-\omega}=1-\left(F_{\epsilon}-F_{\epsilon-\omega}\right) B(\omega / 2 T)$ with $B(x)=\operatorname{coth}(x)$ allows to evaluate the $\epsilon$-integrations in Eqs. (1516) with the result

$$
\begin{aligned}
& \int d \epsilon \epsilon \partial_{T}\left(F_{\epsilon} F_{\epsilon-\omega}\right)=-\omega^{2} \partial_{T} B\left(\frac{\omega}{2 T}\right), \\
& \int d \epsilon \epsilon F_{\epsilon} \partial_{T} F_{\epsilon-\omega}=-\frac{2 \pi^{2} T}{3} \partial_{\omega}\left[\omega B\left(\frac{\omega}{2 T}\right)\right]+\frac{\omega^{3}}{3 T} \partial_{\omega} B\left(\frac{\omega}{2 T}\right) .
\end{aligned}
$$

From Eqs. (17) and (18) we observe that $\delta \mathbf{j}_{Q}^{a}$ is dominated by diffusive modes of frequency $|\omega|<T$, whereas modes with frequencies $|\omega|>T$ give the dominant contribution to $\delta \mathbf{j}_{Q}^{b}$. To appreciate the role played by the different frequency ranges we begin by evaluating the current in two dimensions. The retarded component of the dynamically screened Coulomb interaction reads

$$
V^{R}(\mathbf{q}, \omega) \approx \frac{1}{2 N_{0}} \frac{\kappa_{2 d}}{q} \frac{-\mathrm{i} \omega+D q^{2}}{-\mathrm{i} \omega+D \kappa_{2 d} q},
$$

where $\kappa_{2 d}=4 \pi e^{2} N_{0}$ is the screening vector in two dimensions. By considering first $\delta \mathbf{j}_{Q}^{b}$ one notices that the momentum integration to be performed is identical to the momentum integral in the correction to the electrical conductivity ${ }^{4.5 .6 .7}$, i.e., the integration is logarithmically divergent in the ultraviolet and must be cutoff with the diffusive condition $D q^{2} \tau<1$. In the $\omega$-integration there is a minor difference at low frequencies $|\omega|<T$ due to the second term on the right-hand side of Eq. (18). In two dimensions, with logarithmic accuracy, this difference is negligible and one has

$$
\delta \mathbf{j}_{Q}^{b} \approx \frac{\pi^{2}}{3} \frac{T}{e^{2}} \delta \sigma(-\nabla T),
$$

where $\delta \sigma=-e^{2} /\left(2 \pi^{2}\right) \ln (1 / T \tau)$ is the interaction correction to the electrical conductivity and $T \tau<1$. The other contribution to the thermal current, $\delta \mathbf{j}_{Q}^{a}$, does not depend on the ultraviolet cutoff $1 / \tau$,

$$
\begin{aligned}
\delta \mathbf{j}_{Q}^{a} \approx & -D \nabla T \int_{0}^{T} \frac{d \omega}{2 \pi} \omega \int \frac{d^{2} q}{(2 \pi)^{2}} \\
& \times \operatorname{Im}\left(\frac{1}{-\mathrm{i} \omega+D q^{2}} \frac{\kappa_{2 d}}{q} \frac{1}{-\mathrm{i} \omega+D q \kappa_{2 d}}\right)
\end{aligned}
$$

since the temperature acts as an upper cutoff in the frequency integration. In contrast, in the limit of good metallic screening when $\kappa_{2 d} \rightarrow \infty$, the integration becomes infared divergent. By combining the two contributions we finally write the expression for the thermal conductivity in a form which shows that, although the Wiedemann-Franz law is violated,

$$
\kappa=\frac{\pi^{2}}{3} \frac{k_{B}^{2} T}{e^{2}}\left(\sigma+\delta \sigma+\frac{1}{2} \frac{e^{2}}{\pi h} \ln \left(\hbar D \kappa_{2 d}^{2} / k_{B} T\right)\right),
$$

the integration of diffusive modes in the region $T<$ $D q^{2}, \omega<\tau^{-1}$ yields the same scaling equations for $\sigma$ and $\kappa$,

$$
\frac{d \ln \sigma}{d \ln l}=\frac{d \ln \kappa}{d \ln l},
$$

so that the apparent discrepancies in the literature are no contradiction.

We observe that in the last term of Eq. (22), responsible for the violation of the Wiedemann-Franz law, only the extreme long wavelength modes of the dynamically screened Coulomb interaction with $D q^{2}<|\omega|<T$ are relevant, cf. Eqs. (15) and (17). It has been shown in Ref $\frac{19}{19}$ that these can be summed to all orders and in the end modify the Green's function like a gauge factor,

$$
\check{g}_{t_{1} t_{2}}(\mathbf{x} ; \hat{\mathbf{p}})=e^{\mathrm{i} \check{\varphi}\left(\mathbf{x}, t_{1}\right)} \check{g}_{0 t_{1} t_{2}}(\mathbf{x} ; \hat{\mathbf{p}}) e^{-\mathrm{i} \breve{\varphi}\left(\mathbf{x}, t_{2}\right)},
$$

where $\partial_{t} \check{\varphi}(\mathbf{x}, t)=e \check{\phi}(\mathbf{x}, t)$. Whereas the gauge factors drop in the expression for the electrical current, i.e. the long wavelength modes of the Coulomb interaction do not modify the electrical conductivity, they survive in the heat current to yield

$$
\begin{aligned}
\mathbf{j}_{Q} & =-\frac{N_{0}}{2} \int \frac{d \hat{\mathbf{p}}}{\Omega_{d}} v_{F} \hat{\mathbf{p}} \int d \epsilon\left\{\epsilon g_{0}^{K}(\mathbf{x}, t ; \hat{\mathbf{p}}, \epsilon)\right. \\
& \left.-\frac{1}{2} e(\langle\check{\phi}(t) \check{g}(\mathbf{x}, t ; \hat{\mathbf{p}}, \epsilon)+\check{g}(\mathbf{x}, t ; \hat{\mathbf{p}}, \epsilon) \check{\phi}(t)\rangle)^{K}\right\} .
\end{aligned}
$$

Eq. (25) makes clear the physical origin of the violation of the Wiedemann-Franz law. While the first term on the right-hand side reproduces the non-interacting contribution to the thermal current, the second may be interpreted as the effect of the time dependent fluctuations of the quasi-particle energy in the presence of an electromagnetic environment. Indeed, the extra heat current is proportional to the correlation of voltage and current fluctuations in the system, $\delta \mathbf{j}_{Q}=\left\langle\phi_{1}(\mathbf{x}, t) \delta \mathbf{j}_{e}(\mathbf{x}, t)\right\rangle$, which then leads to the strikingly simple result

$$
\mathbf{j}_{Q}=\frac{\pi^{2}}{3} \frac{k_{B}^{2} T}{e^{2}} \sigma(-\nabla T)-\frac{1}{2} \sigma \nabla\left\langle\phi_{1}(\mathbf{x}, t) \phi_{1}(\mathbf{x}, t)\right\rangle .
$$

Notice that due to the linear current-voltage characteristics of the system under consideration only the first order in the Coulomb interaction contributes to the heat current. By using the fluctuation dissipation theorem (or 
equivalently Eq. (13))

$$
\begin{aligned}
\left\langle\phi_{1}(\mathbf{x}, t) \phi_{1}(\mathbf{x}, t)\right\rangle & =-\frac{1}{e^{2}} \int \frac{d \omega}{2 \pi} B\left(\frac{\omega}{2 T(\mathbf{x})}\right) \sum_{q} \operatorname{Im} V^{R}(\mathbf{q}, \omega) \\
& =\int \frac{d \omega}{2 \pi} B\left(\frac{\omega}{2 T(\mathbf{x})}\right) \omega \operatorname{Re} Z(\omega)
\end{aligned}
$$

direct contact can be made with the conventional perturbation theory, i.e. with $\delta \mathbf{j}_{Q}^{a}$ in Eq. (15).

Instead of parameterizing the local voltage fluctuations in terms of an interaction $V^{R}(\mathbf{q}, \omega)$ we will in the following parameterize them in terms of the impedance of the local electromagnetic environment, $Z(\omega)$. By doing so, the thermal conductivity reads

$\kappa=\frac{\pi^{2}}{3} \frac{k_{B}^{2} T}{e^{2}} \sigma+\frac{\sigma k_{B}}{e^{2}} \int d E\left(\frac{E / 2 k_{B} T}{\sinh \left(E / 2 k_{B} T\right)}\right)^{2} \frac{\operatorname{Re} Z(E / \hbar)}{h / e^{2}}$,

where for clarity we put back the $\hbar$ and $k_{B}$. We will now discuss three different examples for the impedance $Z$. The simplest situation consists of a purely ohmic environment where $Z(E / \hbar)=R$. The thermal conductivity is found linear in the temperature, strong deviations from the Wiedemann Franz law are found when the environmental resistance is of the order of the resistance quantum $h / e^{2}$ or larger. The explicit result is

$$
\kappa=\frac{\pi^{2}}{3} \frac{k_{B}^{2} T}{e^{2}} \sigma\left(1+2 R / h / e^{2}\right) .
$$

From the retarded Coulomb interaction as given in Eq. (19) we determine the impedance of a thin film as $\operatorname{Re} Z(E / \hbar)=(1 / 4 \pi \sigma) \ln \left(\hbar D \kappa_{2 d}^{2} k_{B} T / E^{2}\right)$. Due to the weak logarithmic energy dependence of the impedance the thermal conductivity is to good accuracy obtained from Eq. (29) with $R=\operatorname{Re} Z\left(k_{B} T / \hbar\right)$. As a third example we consider a $R C$-transmission line, as a model of a gated wire. The impedance is $\operatorname{Re} Z=\frac{1}{2} \sqrt{R_{0} / 2|\omega| C_{0}}$, where $R_{0}$ and $C_{0}$ are the resistance and capacitance per unit length. We find a contribution to the thermal conductivity which is proportional to the square root of the temperature,

$$
\kappa=\frac{\pi^{2}}{3} \frac{k_{B}^{2} T}{e^{2}} \sigma+2.456 \frac{k_{B}}{e^{2}} \sigma \frac{\sqrt{\hbar k_{B} T R_{0} / C_{0}}}{h / e^{2}},
$$

with 2.456 the approximate numerical value for $3 \zeta(3 / 2) \Gamma(3 / 2) / 2^{3 / 2}$.

In summary we calculated the thermal conductivity of disordered metals. In the two-dimensional electron system the scaling equations for the thermal and the electrical conductivity are the same, nevertheless the Wiedemann-Franz law does not hold. The deviations from the Wiedemann-Franz law are comparable in size to the localization effects. It is interesting to note that this is in qualitative agreement with observations made in the cuprates ${ }^{3.20}$ : The resistivity of PCCO in Ref $\frac{3}{\underline{3}}$ shows a well pronounced low temperature anomaly which has been attributed to localization effects, and at the same time the low temperature heat conductivity is larger than what would be expected from the WiedemannFranz law. In the low temperature resistivity of Tl2201 in Ref ${ }^{20}$ no indications of localization effects are seen and the Wiedemann-Franz law is perfectly obeyed within the experimental accuracy. Quantitatively on the other hand the agreement of our theory with Ref ${ }^{3}$ remains poor, since the sheet resistance was estimated as $R_{\square} \approx h /\left(60 e^{2}\right)$ from which we expect a much smaller enhancement of the heat conductivity than observed experimentally. By measuring the Lorenz ratio in a gated film or wire as function of the gate capacitance, it should be possible to test our predictions experimentally.

R.R. and G.S. acknowledge partial financial support from MIUR under grant COFIN2002022534. We acknowledge valuable discussions with U. Eckern, C. Castellani, and C. Di Castro.
1 G. V. Chester and A. Thellung, Proc. Phys. Soc. London 77, 1005 (1961).

2 J. S. Langer, Phys. Rev. 128, 110 (1962).

3 R. W. Hill et al, Nature 414, 711 (2001).

4 B. L. Altshuler and A. G. Aronov, Sov. Phys. JETP 50, 968 (1979).

5 B. L. Altshuler and A. G. Aronov, Solid State Communications 46, 429 (1983).

6 A. M. Finkelstein, Zh. Eksp. Teor. Fiz. 84, 168 (1983); Sov. Phys. JETP 57, 97 (1983).

7 C. Castellani, C. DiCastro, P. A. Lee, and M. Ma, Phys. Rev. B 30, 527 (1984).

8 C. Castellani, C. DiCastro, P. A. Lee, M. Ma, S. Sorella, and E. Tabet, Phys. Rev. B 33, 6169 (1986).

9 C. Castellani and C. DiCastro, Phys. Rev. B 34, 5935 (1986).

10 C. Castellani, C. DiCastro, G. Kotliar, P. A. Lee, and G.
Strinati, Phys. Rev. Lett. 59, 477 (1987).

11 D. Livanov, M. Yu. Reizer, and A. V. Sergeev, Sov. Phys. JETP 72, 760 (1991).

12 D. R. Niven and R. A. Smith, cond-mat/0301451

13 Non-equilibrium Superconductivity, edited by D. N. Langenberg and A. I. Larkin (North-Holland, Amsterdam, 1986).

14 C. J. Lambert and R. Raimondi, J. Phys.: Condens. Matter 10, 901 (1998).

15 P. Schwab and R. Raimondi, Ann. Physik (Leipzig) 12, 471 (2003).

16 J. Rammer and H. Smith, Rev. Mod. Phys. 58, 323 (1986).

17 G. Eilenberger, Z. Phys. 214, 195 (1968).

18 K. D. Usadel, Phys. Rev. Lett. 25, 507 (1970).

19 P. Kopietz, Phys. Rev. Lett. 81, 2120 (1998).

20 C. Proust et al, Phys. Rev. Lett. 89, 147003 (2002). 